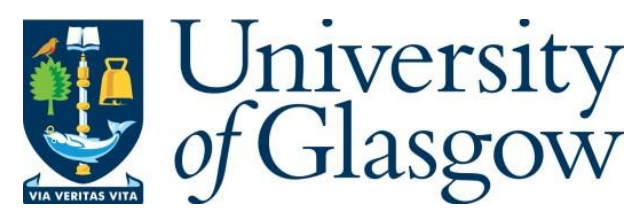

Gallacher, K. et al. (2017) Mid-infrared n-Ge on Si Plasmonic Based Microbolometer Sensors. In: 2017 IEEE 14th International Conference on Group IV Photonics (GFP), Berlin, Germany, 23-25 Aug 2017, pp. 3-4. ISBN 9781509065684.

There may be differences between this version and the published version. You are advised to consult the publisher's version if you wish to cite from it.

http://eprints.gla.ac.uk/152542/

Deposited on: 27 November 2017

Enlighten - Research publications by members of the University of Glasgow http://eprints.gla.ac.uk 


\title{
Mid-Infrared n-Ge on Si Plasmonic Based Microbolometer Sensors
}

\author{
Kevin Gallacher*, Ross W. Millar*, Valeria Giliberti ${ }^{\dagger}$ Eugenio Calandrini ${ }^{\dagger}$ Leonetta Baldassarre ${ }^{\dagger}$ Jacopo $^{*}$ Frigerio $^{\ddagger}$ \\ Andrea Ballabio ${ }^{\ddagger}$ Emilie Sakat $^{\S}$ Giovanni Pellegrini $^{\S}$ Giovanni Isella $^{\ddagger}$ Michele Ortolani $^{\dagger}$ \\ Paolo Biagioni ${ }^{\S}$ and Douglas J. Paul*, \\ ${ }^{*}$ School of Engineering, University of Glasgow, Rankine Building, Oakfield Avenue, Glasgow G12 8LT, United Kingdom \\ ${ }^{\dagger}$ Dipartimento di Fisica, Sapienza Universitá di Roma, Piazzale Aldo Moro 5, I-00185 Rome, Italy \\ ${ }^{\ddagger}$ L-NESS, Dipartimento di Fisica, Politecnico di Milano, Polo di Como, Via Anzani 42, I-22100 Como, Italy \\ $\S$ Dipartimento di Fisica, Politecnico di Milano, Piazza Leonardo da Vinci 32, I-20133 Milano, Italy \\ IDouglas.Paul@glasgow.ac.uk
}

\begin{abstract}
The detection and amplification of molecular absorption lines from a chemical weapons simulant is demonstrated using plasmonic antennas fabricated from n-Ge epitaxially grown on $\mathrm{Si}$. A free-standing $\mathrm{Si}_{0.25} \mathbf{G e}_{0.75}$ microbolometer detector with n-Ge plasmonic antenna is demonstrated as an integrated midinfrared plasmonic sensor.
\end{abstract}

\section{INTRODUCTION}

Plasmons are the free electron oscillations in a metal coupled to photons [1] and allow the near field amplification of radiation below the plasmon edge defined in wavelength as

$$
\lambda_{p}=2 \pi c \sqrt{\frac{\epsilon_{0} \epsilon_{\infty} m^{*}}{q^{2} N}}
$$

where $N$ is the doping density, $m^{*}$ is the effective mass, $\epsilon_{0}$ is the permittivity of free space, $\epsilon_{\infty}$ is the high frequency dielectric constant, $q$ is the electron charge, $c$ is the speed of light and $\lambda$ is the wavelength [2]. In the visible, metals such as $\mathrm{Au}$ and $\mathrm{Ag}$ can be used but these are incompatible with silicon foundries due to fast diffusion and deep level trap states. Here we demonstrate heavily doped semiconductors, doped well above the Mott metal-insulator transition criteria, as plasmonic materials for the mid-infrared wavelengths. Many molecules can be identified at mid-infrared wavelengths due to the strong molecular absorption lines related to individual bonds. We demonstrate n-Ge epitaxially grown on Si substrates and use it as a mid-infrared plasmonic material. Plasmonic antennas are demonstrated to provide amplification of specific molecular absorption lines of a chemical weapon simulant as an example. Applications of mid-infrared plasmonic sensors include healthcare, environmental and chemical, biological, radiological, nuclear and explosives (CBRNE) security sensing.

n-Ge epilayers on high resistivity $\mathrm{Si}$ (001) substrates were grown using low-energy plasma enhanced chemical vapour deposition [3]. The decrease in the wavelength (increase in the wavenumber) of the plasmon edge as the doping density increased for n-Ge epilayers of thicknesses between $600 \mathrm{~nm}$ and $1000 \mathrm{~nm}$ [2][4][5] is demonstrated in Fig. 1. An activated doping density of $1.3 \times 10^{20} \mathrm{~cm}^{-3}$ at $300 \mathrm{~K}$ enables the lowest wavelength of $\sim 3.9 \mu \mathrm{m}$ to be achieved. This is sufficient to

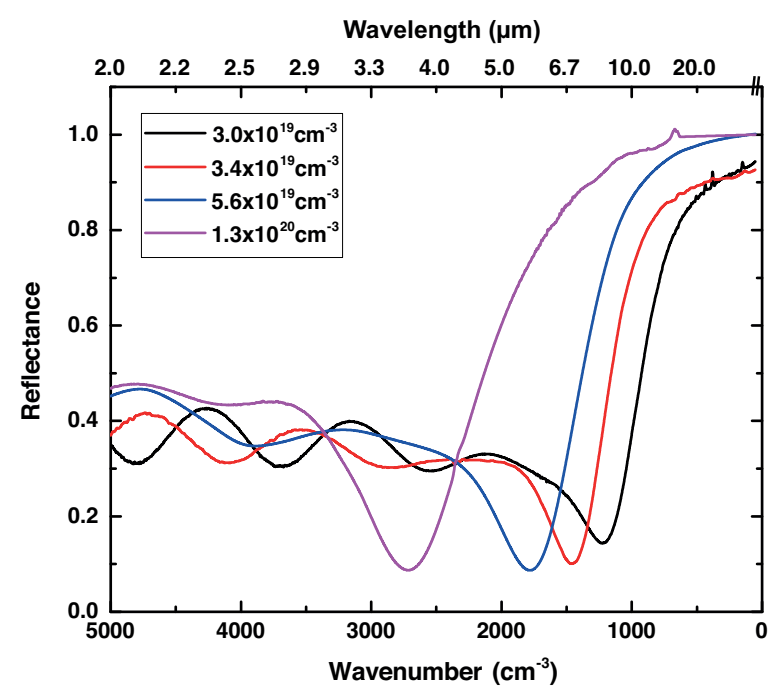

Fig. 1. The reflectance of 4 different $n-G e$ doped layers all with thickness between $600 \mathrm{~nm}$ and $1000 \mathrm{~nm}$ at $300 \mathrm{~K}$.

enable the whole of the important 6.7 to $20 \mu \mathrm{m}$ molecular fingerprint region which has only really been successfully used with Fourier transform infrared (FTIR) systems and a significant part of the 3 to $5 \mu \mathrm{m}$ gas detection window.

Electron beam lithography and reactive ion etching [6] on the $1000 \mathrm{~nm}$ thick n-Ge material (comparable to the skin depth in the 10 to $20 \mu \mathrm{m}$ wavelength region) [4] was used to fabricate a range of single and gap antennas into arrays over a $5 \times 5$ $\mathrm{mm}^{2}$ array. Fig. 2 provides a scanning electron microscope (SEM) image of a set of gap antennas. The substrate side plasmonic resonance can be tuned by varying the antenna length ( 1 to $4 \mu \mathrm{m})$ allowing the targeting of specific molecular absorption lines for identification [4].

Fig. 3 demonstrates a chemical weapons application using the n-Ge antennas in Fig. 2. The FTIR spectrum of the mustard gas simulant chloroethyl methyl sulphide (CEMS - see Fig. 3 insert for chemical structure) has a $\mathrm{C}-\mathrm{Cl}$ bond absorption doublet at approximately $14 \mu \mathrm{m}$ wavelength. Most explosives, 


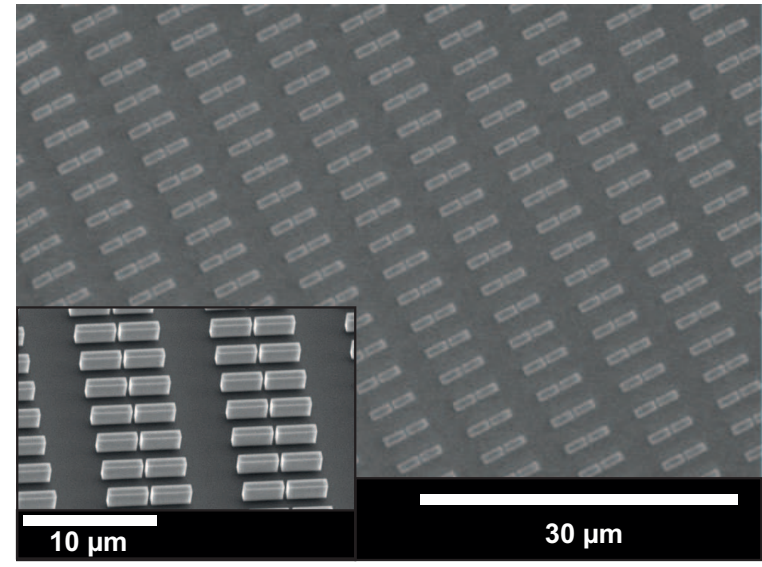

Fig. 2. SEM images of an array of gap antennas with $3 \mu \mathrm{m}$ long $\mathrm{n}-\mathrm{Ge}$ sections and $300 \mathrm{~nm}$ gaps fabricated on top of a Si substrate.

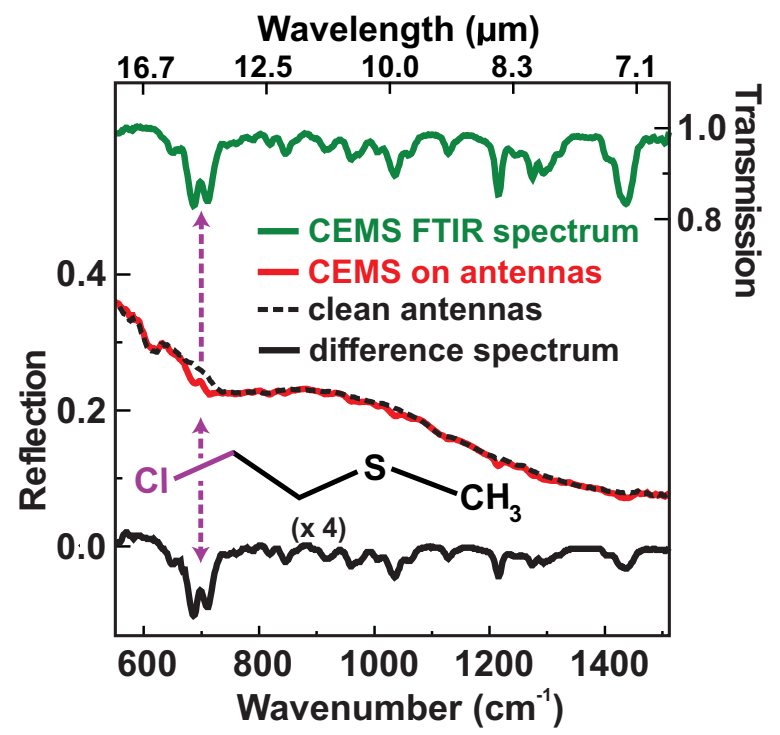

Fig. 3. Top: the transmission spectrum of CEMS measured by FTIR. Middle: the reflection spectrum of a n-Ge plasmonic antenna array without CEMS (dashed black line) and the array with CEMS (red line). Bottom: the difference between the clean and CEMS coated plasmonic antenna spectrums.

chemical and biological weapons have unique molecular bonding absorption lines in the spectroscopic fingerprint region of 6.7 to $15 \mu \mathrm{m}$. A few drops of CEMS liquid was deposited on a set of antennas (red line in Fig. 3) and the difference was taken from a clean set of antennas (black dashed line) to leave the difference spectrum of CEMS (solid black line at bottom of Fig. 3). Only the absorption lines for wavelengths longer than the plasmon edge $\left(10 \mu \mathrm{m}\right.$ for this material doped at $2.3 \times 10^{19}$ $\mathrm{cm}^{-3}$ ) are amplified by the plasmonic antennas. $\mathrm{n}-\mathrm{Ge}$ antennas used to measure absorptions in polydimethylsiloxane (PDMS) have demonstrated amplification up to two orders of magnitude at $12.5 \mu \mathrm{m}$ wavelength [4]. Whilst $\mathrm{Au}$ and $\mathrm{Ag}$ do provide lower losses than $\mathrm{n}-\mathrm{Ge}$ due to the higher electrical conductivity, this is counterbalanced by a significantly higher degree of field confinement in $\mathrm{n}-\mathrm{Ge}$ since the doped semiconductor

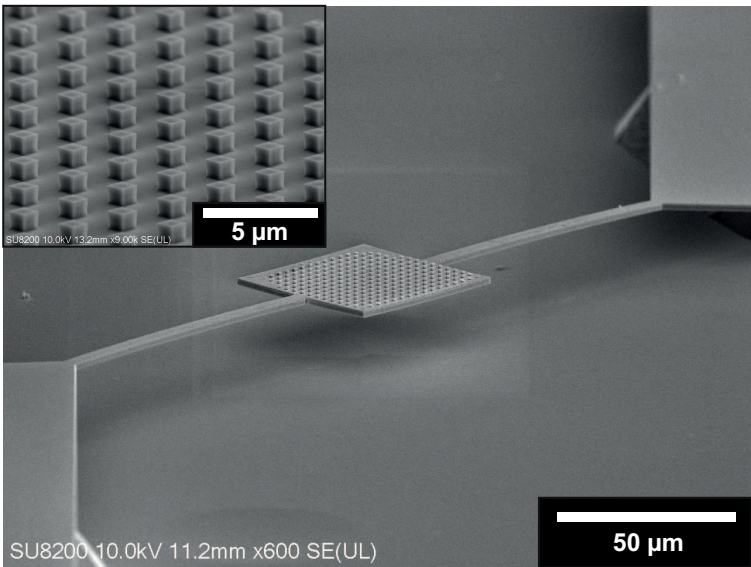

Fig. 4. A SEM image of a microbolometer sensor with single plasmonic antenna array to provide amplification for detection of molecular absorption at specific wavelengths. The insert provides a detailed SEM image of the plasmonic antenna array.

demonstrates true plasmonic behaviour [5].

Fig. 4 is a SEM image of single $\mathrm{n}-\mathrm{Ge}$ antenna fabricated on a free standing microbolometer detector. Simulations indicate that a $1 \mu \mathrm{W}$ absorption from a plasmonic resonance illuminated from a mid-infrared source raises the microbolometer temperature by $5.5 \mathrm{~K}$ inside $150 \mathrm{~ms}$ whilst the microbolometers have sensitivity to $\mathrm{mK}$ temperature changes.

\section{CONCLUSION}

Physical doping has been used to demonstrate plasmonic behaviour in $\mathrm{Ge}$ antenna on $\mathrm{Si}$ substrates down to $\sim 3.9 \mu \mathrm{m}$ wavelengths. Examples of chemical weapons identification and integrated mid-infrared sensors on a Si platform using microbolometer detectors have been demonstrated.

\section{ACKNOWLEDGMENT}

The research leading to these results has received funding from the European Unions Seventh Framework Programme under grant agreement no. 613055 .

\section{REFERENCES}

[1] A. Boltasseva and H. A. Atwater, "Low-loss plasmonic metamaterials," Science, vol. 331, no. 6015, pp. 290-291, 2011. [Online]. Available: http://science.sciencemag.org/content/331/6015/290

[2] P. Biagioni et al., "Group-IV midinfrared plasmonics," $J$. Nanophotonics, vol. 9, no. 1, p. 093789, 2015. [Online]. Available: http://dx.doi.org/10.1117/1.JNP.9.093789

[3] G. Isella et al., "Low-energy plasma-enhanced chemical vapor deposition for strained $\mathrm{Si}$ and $\mathrm{Ge}$ heterostructures and devices," Solid-State Electron., vol. 48, no. 8, pp. 1317-1323, Aug. 2004. [Online]. Available: http://linkinghub.elsevier.com/retrieve/pii/S0038110104000565

[4] L. Baldassarre et al., "Mid-infrared plasmon-enhanced spectroscopy with germanium antennas on silicon substrates," Nano Lett., vol. 15, no. 11, pp. 7225-7231, 2015. [Online]. Available: http://dx.doi.org/10.1021/acs.nanolett.5b03247

[5] J. Frigerio et al., "Tunability of the dielectric function of heavily doped germanium thin films for mid-infrared plasmonics," Phys. Rev. B, vol. 94, p. 085202, Aug 2016. [Online]. Available: http://link.aps.org/doi/10.1103/PhysRevB.94.085202

[6] M. M. Mirza et al., "Determining the electronic performance limitations in top-down-fabricated si nanowires with mean widths down to $4 \mathrm{~nm}$,' Nano Lett., vol. 14, no. 11, pp. 6056-6060, 2014. [Online]. Available: http://dx.doi.org/10.1021/n15015298 